\title{
A correlational study of systemic blood pressure and intraocular pressure in a young South African adult population*
}

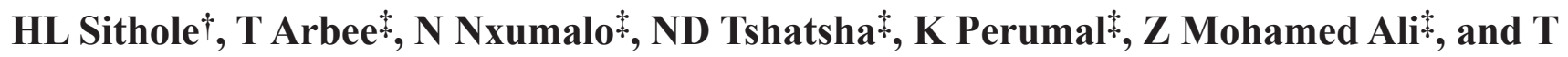 \\ Souza*
}

$\dagger$ University of South Africa, Academic and Research Portfolio, Research Directorate, PO Box 392, UNISA, 0003 South Africa

$\$$ University of KwaZulu-Natal, School of Physiotherapy, Sport Science and Optometry, Discipline of Optometry, P/Bag X 54001, Durban, 4000 South Africa

$\uparrow<$ sithohl@unisa.ac.za>

Received 20 January 2009; revised version accepted 27 October 2009

\begin{abstract}
Increased blood pressure (BP) and raised intraocular pressure (IOP) are probably both common occurrences among the South African population. If left untreated both conditions have detrimental complications. Previous cross-sectional studies suggested BP was positively related to IOP. This study therefore sets out to determine in a young South African adult population the correlation between systemic BP and IOP. Systemic BP was measured using an electronic sphygmomanometer and IOP using a Goldman applanation tonometer. For all subjects, two averages were obtained from three measurements each of BP and IOP. Other clinical procedures such as uncompensated visual acuity (VA), pinhole and direct ophthalmoscopy were done to exclude underlying factors possibly affecting either BP or IOP before the commence-
\end{abstract}

ment of the investigation. Two hundred $(N=200)$ subjects were included in the study and their ages ranged from 18 to 30 years with a mean of $21 \pm$ 3.9 years. The correlation coefficients between average IOP and average systolic or diastolic BP respectively were 0.67 and 0.55 . These weak positive correlations suggested that with an increase in BP there is a corresponding increase in IOP. Also, similar correlation between IOP and BP was found to exist amongst both males and females with systolic $\mathrm{BP}$ having a greater effect. This study validates the importance of evaluating either systemic BP or IOP amongst all patients seen by primary eye-care practitioners, and that such evaluations should form part of daily routine patient examination.

Key words: Blood pressure (BP), intraocular pressure (IOP), correlation research, glaucoma

\footnotetext{
${ }^{\dagger}$ BOptom MOptom

\$BOptom
}

*This paper was prepared as part of a Research Publication course undertaken by fourth year students at the Univer-
sity of KwaZulu-Natal under the supervision of HL Sithole. 


\section{Introduction}

An abnormally high blood pressure (BP) is a major cause of morbidity and mortality in the Western countries $^{1}$ including South Africa ${ }^{2}$, and is one of the most common clinical conditions requiring long term medical care ${ }^{3}$. Hypertension is characterized by a persistent systolic BP of more than $140 \mathrm{mmHg}$ and a diastolic BP of more than $90 \mathrm{mmHg} .{ }^{4}$ Increased BP can result in symptoms such as dizziness, headaches as well as more serious complications such as coronary artery disease, heart failure or even death ${ }^{5}$.

Hypertension is also associated with ocular complications such as optic neuropathy and hypertensive retinopathy which is one of the leading causes of blindness in developing countries ${ }^{6}$. Similarly, an abnormally increased intraocular pressure (IOP), which is known to be a glaucoma risk factor ${ }^{7,8}$, causes permanent damage to the optic nerve leading to vision impairment $^{3}$. Glaucoma affects more than 66 million people worldwide and as a result at least 6.8 million individuals have bilateral blindness ${ }^{3}$. Therefore, both high BP and IOP should be given adequate attention by primary eye-care practitioners including optometrists. This suggests the need for optometrists and other eye-care practitioners to routinely measure BP and IOP on their patients.

Several cross-sectional studies of Western populations have shown a positive relationship between systemic $\mathrm{BP}$ and $\mathrm{IOP}^{3,9,10}$. McLeod et al. ${ }^{11}$ found that change in IOP was positively correlated with change in systolic BP. Similarly, Leshe and Podgor ${ }^{12}$ found a significant positive correlation between IOP and BP. In people aged between 43-86 years, living in Beaver Dam, Wisconsin (USA), a longitudinal study ${ }^{13}$ of systemic BP, IOP, and history of use of some BP medications revealed that intraocular pressures were significantly correlated with systolic and diastolic blood pressures at both baseline and follow up. Although McLeod et al. ${ }^{11}$ found no consistent relationship between IOP and BP initially in their study, when an autoregressive model was used to examine the relationship between change in BP and IOP after one or two years, a change in IOP was positively correlated to change in systolic BP. The results indicated that changes in IOP over time are associated with changes in systolic BP.
The aim of this cross-sectional study was therefore to investigate in a young South African adult population the relationship between systemic blood pressure and intraocular pressure.

\section{Methods}

This was a cross-sectional study based on a theoretical framework where convenience sampling method was employed. Ethical approval was obtained from the University of KwaZulu-Natal Ethics Committee before the commencement of the study. All subjects were University of KwaZulu-Natal students. They were all briefly informed about the nature and purpose of the study. Those who agreed to participate were given the University of KwaZulu-Natal consent form for completion before the commencement of the study. All those requested to participate in the study completed the form, therefore, nobody was excluded from the study as a result of refusal to complete the form. A short case history was taken for each subject and subsequently ophthalmic tests such as uncompensated visual acuity (VA), pinhole test, and direct ophthalmoscopy were used to assess their oculo-visual status. This process was done to exclude subjects with underlying factors possibly affecting BP and IOP. If no underlying factors were found, subjects were subsequently subjected to sphygmomanometry and contact tonometry for BP and IOP measurements. Three measurements were done for each test and the average value was then used in the study.

Descriptive statistics were used to analyse the results using the Statistical Package for Social Sciences (SPSS) computer programme. A one-sample Kolmogorov-Smirnov test was done to ensure that the data was normally distributed and relationships between BP and IOP were determined using Pearson correlation coefficients.

\section{Results}

A total of 200 University of KwaZulu-Natal students were included in the study with males and females being equally distributed. All race groups were included and their ethnic distribution is shown in Figure 1. 


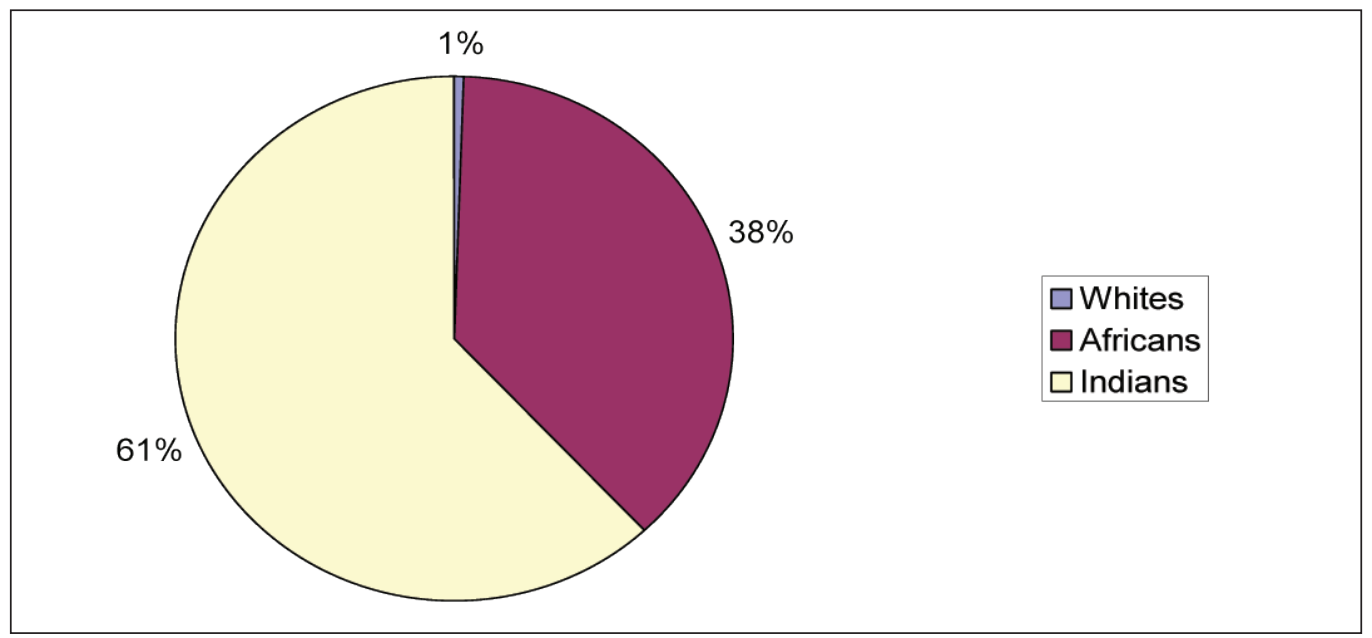

Figure 1: Showing the race distribution of the subjects. The majority $(61 \%)$ of the subjects were Indians.

Their ages ranged from 18 to 30 years with a mean of $21 \pm 3.9$ years. The most common age group, which was 19 to 22 years accounted for $90 \%$ of the 200 participants. Only a few were 25 years or older. The distribution of the ages of the participants is shown in Figure 2.

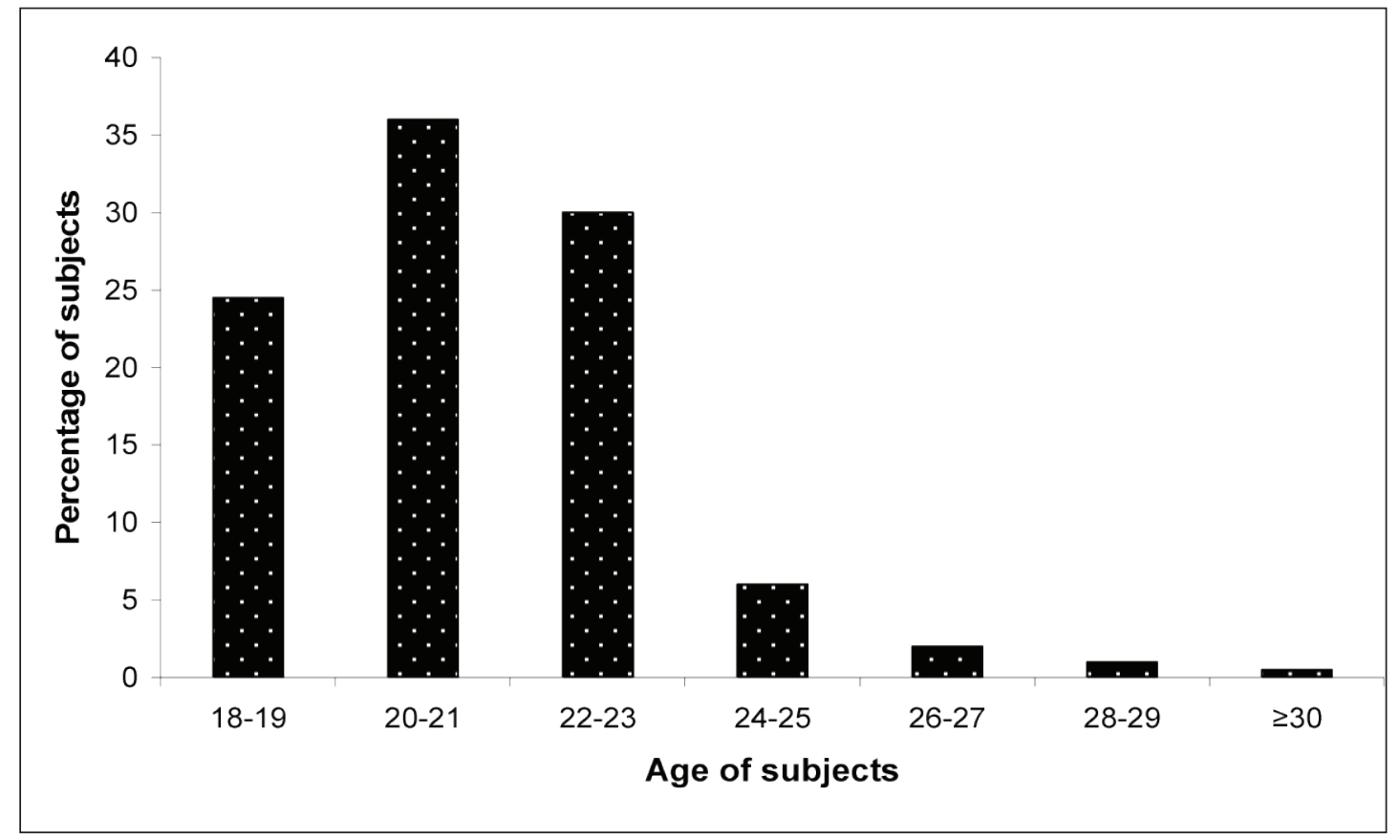

Figure 2: The age distribution of the subjects included in the study.

A one-sample Kolmogorov-Smirnov Test (a non-parametric test) was done for average values of IOP, systolic BP and diastolic BP and it was found that data was normally distributed $(p>0.05)$.

The Pearson correlation coefficient for average IOP and systemic BP was 0.67 with a probability value of $<0.05$. With an increase in average systolic BP there is an increase in the average IOP. The relationship between average IOP and average systolic BP is shown in Figure 3. 


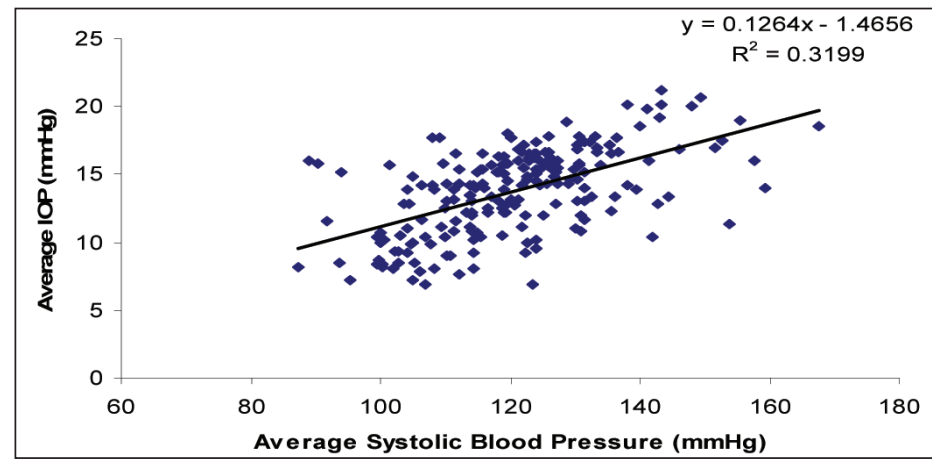

Figure 3: Showing the relationship between average IOP and average systolic BP.

For average IOP and average diastolic BP Pearson correlation reveals a probability value of $<0.05$. With an increase in average diastolic BP there is an increase in the average IOP, with a correlation coefficient of 0.55 . The relationship between average IOP and average diastolic BP is shown in Figure 4.

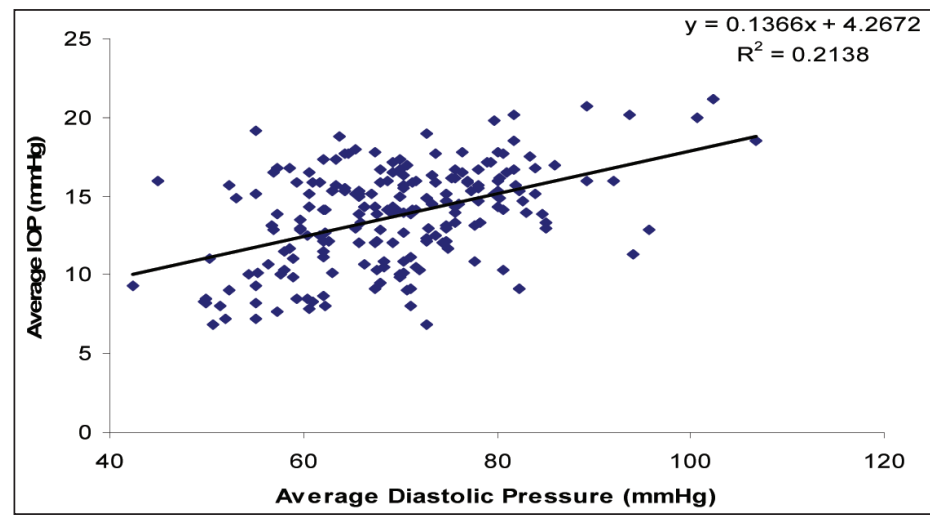

Figure 4: Relationship between average IOP and average diastolic BP.

The Pearson correlation coefficient was 0.68 with a probability value of $<0.05$ for average IOP and average systolic BP in males. With an increase in average systolic BP there is an increase in the average IOP. The relationship between average IOP and average systolic BP in males is shown in Figure 5.

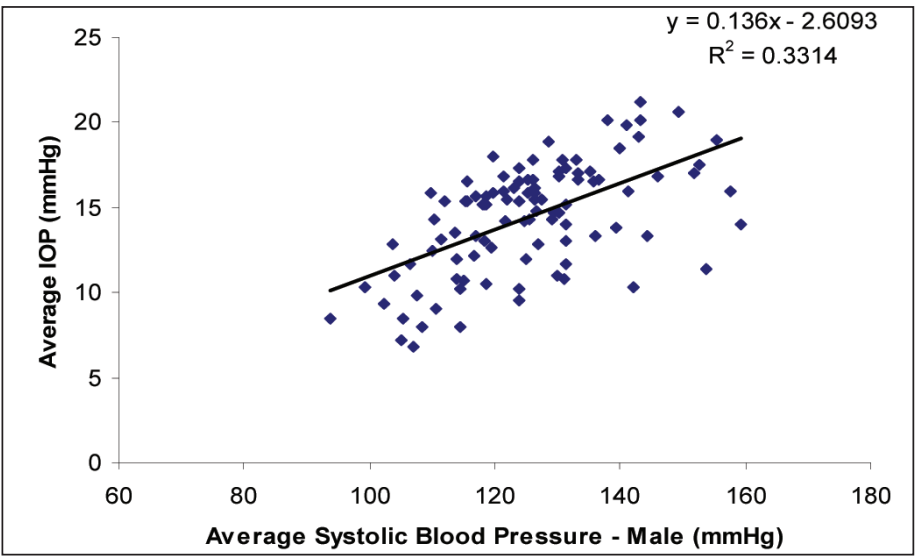

Figure 5: Relationship between average IOP and average systolic BP in males.
The Pearson correlation coefficient was 0.56 with a probability value of $<0.05$ for average IOP and average diastolic BP in males. The relationship between average IOP and average diastolic BP in males is shown in Figure 6.

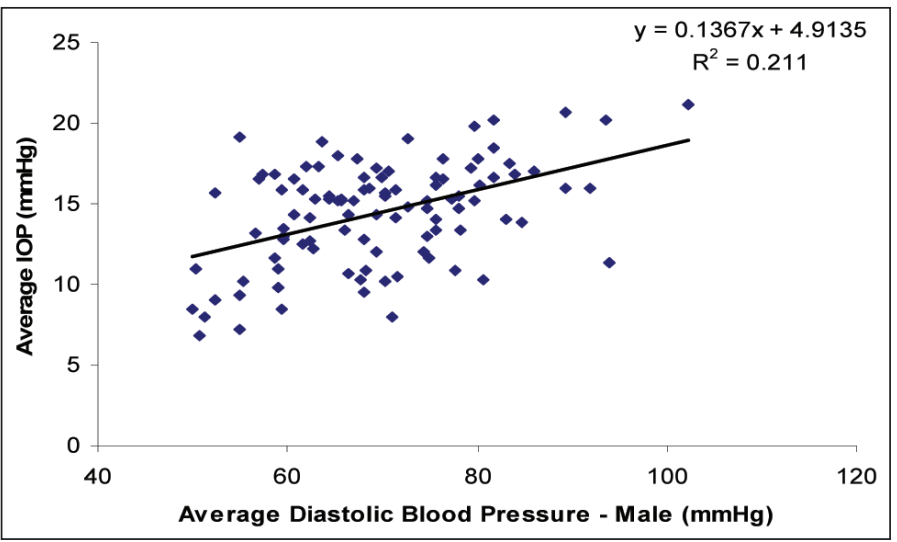

Figure 6: Relationship between average IOP and average diastolic BP in males.

The Pearson correlation coefficient was 0.58 with a probability value of $<0.05$ for average IOP and average systolic BP in females. With an increase in average systolic BP there is an increase in the average IOP. The relationship between average IOP and average systolic BP in females is shown in Figure 7.

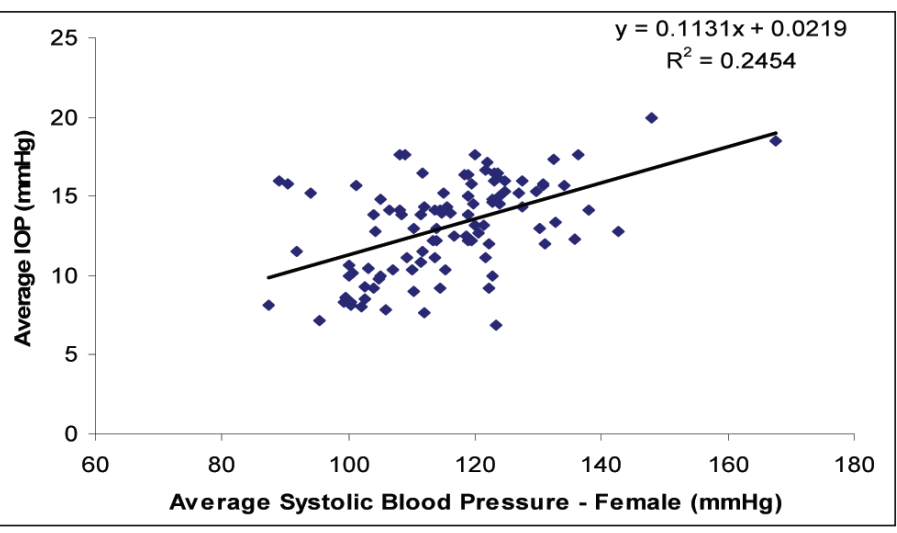

Figure 7: Relationship between average IOP and average systolic BP in females.

The Pearson correlation coefficient was 0.59 with a probability value of $<0.05$ for average IOP and average diastolic BP in females. With an increase in average diastolic BP there is an increase in the average IOP. The relationship between average IOP and average diastolic BP in females is shown in Figure 8. 


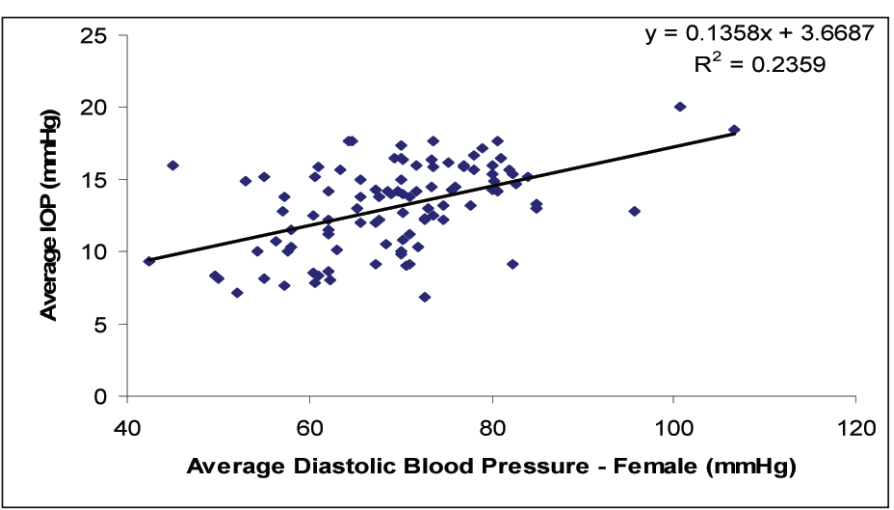

Figure 8: Relationship between average IOP and average diastolic BP in females.

\section{Discussion}

Increased BP and IOP may have adverse effects if left unattended. This suggests the need for patients to be always examined for any BP or IOP changes whenever they visit primary eye-care professionals. This study was therefore carried out to investigate possible relationships between systemic BP and IOP in a young South African adult population.

The sample mostly included Indians (61\%). This could be associated with that mostly Indians have enrolled at the University of KwaZulu-Natal. It will be interesting to see how this demography changes in the future since the university has enacted policies that seek to encourage other race groups to enroll with the University.

Most of the subjects were aged between 19 and 20 years and a one-sample Kolmogorov-Smirnov test revealed normal distribution of data $(p>0.05)$. Pearson correlation coefficients (a parametric test) were used to determine correlations between average IOP, average systolic BP and average diastolic BP. A significant correlation was found between average IOP and average systolic $\mathrm{BP}(r=0.67, p<0.05)$.

Figure 3 showed that an increase in average systolic BP resulted in an increase in the average IOP, with a correlation coefficient of 0.6 . Figure 4 represents the relationship between average IOP and average diastolic BP. A significant correlation was found $(r=0.55, p<0.05)$, and with an increase in average diastolic BP there is an increase in the average IOP, with a correlation coefficient of 0.5 . The results are corroborated by findings in other studies ${ }^{3,11,12}$. These findings also showed a positive correlation between IOP and BP. This reveals the importance of always taking into cognizance the importance of evaluating either the IOP or BP amongst patients. The findings also highlight the significance of why primary eye care practitioners such as optometrists should incorporate measurements of IOP and BP into their routine patient examinations.

In other different studies ${ }^{14-16}$, it was also found that the systolic BP has a greater effect on the IOP than the diastolic BP. Therefore, this finding agrees with the findings in this study that reveals a higher correlation coefficient for systolic and intraocular pressure. This higher coefficient between the systolic BP and IOP is possibly due to the systolic BP being the maximum arterial pressure exerted by the heart during contraction of the left ventricle ${ }^{4}$. Generally, an increase in the systolic BP will result in an increase in the blood flow to the ciliary body in the eye, which is responsible for the production of aqueous. This increased blood supply will bring about an increase in aqueous production, resulting in an increase in $\mathrm{IOP}^{4}$. The increased IOP will also have an effect on the drainage of the aqueous humour via the episcleral veins by affecting the pressure gradient between the aqueous in the anterior chamber and in the episcleral veins ${ }^{4}$.

A correlation coefficient of 0.7 or greater is usually considered to be a strong correlation. This indicates that although there were positive relationships between IOP and systolic BP, IOP and diastolic BP, they were not particularly strong correlations.

The correlation between IOP and BP was also confirmed between both males and females with probability values of less than 0.05 being found for the correlations between IOP and systolic BP as well as for IOP and diastolic BP. See Figures 5 and 6, where in males, the correlation coefficients for systolic and diastolic BP with IOP were 0.68 and 0.56 respectively. Similarly, amongst females, Figures 7 and 8 also show the positive correlation for both systolic and diastolic BP with IOP. The correlation coefficients in females for systolic (0.58) and diastolic (0.59) with IOP also show that an increase in systolic or diastolic $\mathrm{BP}$ results in an increase in IOP. This therefore further substantiates the existence of the correlation between $\mathrm{BP}$ and IOP and the reason why their measurements should be of paramount importance among primary eye care practitioners such as optometrists. This practice would assist in early detection of any possible ocular manifestations of both increased BP and IOP, 
thus preventing some cases of avoidable blindness.

The correlation coefficient with regard to systolic $\mathrm{BP}$ and IOP were higher in males as compared to females ( 0.68 in males and 0.59 in females). This study suggests the IOP in males is thus more likely to be positively affected by the systolic BP than in females. The diastolic BP correlation with IOP on the other hand was slightly higher in females when compared to the diastolic in males ( 0.59 in females compared to 0.56 in males). Thus diastolic pressure possibly has a greater effect on IOP in females when compared to males. This variation between males and females could be due to various factors such as hormonal influences, physical attributes and general lifestyle differences.

\section{Conclusion and Recommendations}

The study reveals that the relationship between IOP and BP established by previous studies holds in a young South African population. This correlation therefore validates the monitoring of patients presenting with either increased systemic BP or IOP and provides adequate information relating to the importance of evaluating both BP and IOP by primary eye health care practitioners such as optometrists.

It is recommended that future studies should include subjects older than 40 years (since there is greater prevalence of hypertension within this age group) as well as pediatric studies, to investigate if this correlation also exists in children. Also, people of different races may have different diets and lifestyle, therefore further studies on lifestyle factors such as caffeine intake, smoking, or exercise should be conducted to determine their effect on this correlation. Subjects with systemic and ocular pathology should also be included, to verify if the relationship holds.

\section{References}

1. Feeman WE. Prediction of the population at risk of atherothrombotic disease. Exp Clin Cardiol 20049 235-241.

2. Steyn K, Gaziano T, Bradshaw D, Laubscher R, Fourie J. Hypertension in South African adults: results from the demographic and health survey, 1998. J Hyper $2001191717-$ 1725.

3. Perlman JI, Delay CM, Sothern RB, Skolnick KA, Murray D, Jacobs RW, Shue JL, Kaplan E, Friedman NC, Nemchausky BA, Ryan MD, Kanabrocki EL. Relationships between $24 \mathrm{~h}$ observations in intraocular pressure vs blood pressure, heart rate, nitric oxide and age in the medical chronobiology aging project. Clin Ter 2007 158 31-47.

4. Liang Y, Downs JC, Fortune B, Cull GA, Cioffi GA, Wang L. Impact of systemic blood pressure on the relationship between intraocular pressure and blood flow in the optic nerve head of non-human primates. Invest Ophthalmol Vis Sci 200950 2154-2160.

5. Khaw KT, Foster P. The eye - window to the soul or a mirror of systemic health? Or: What weight to give retinopathy as a risk factor for IHD. Heart 200995 348-349.

6. Gillow JT, Gibson JM, Dodson PM. Hypertension and diabetic retinopathy: what's the story? Br J Ophthalmol 1999 83 1083-1087.

7. Luciano Bonomi MD, Giorgio Marchini MD, Michele Marraffa MD, Paolo Bernardi MD, Roberta Morbio MD, Aldo Varotto MD. Vascular risk factors for primary open angle glaucoma: The Egna-Neumarkt study. Br J Ophthalmol 2000107 1287-1293.

8. Van Niekerk M, Van Rooyen FC, Joubert G, Hiemstra LA. The prevalence of the diagnosis of increased intra-ocular pressure in a general practice. SA Fam Pract 20064816.

9. Mori K, Ando F, Nomura H, Sato Y, Shimokata H. Relationship between intraocular pressure and obesity in Japan. Int J Epidemiol 200029 661-666.

10. Lee JS, Lee SH, Oum BS, Chung JS, Cho BM, Hong JW. Relationship between intraocular pressure and systemic health parameters in a Korean population. Clin Exp Ophthalmol $200230237-241$.

11. McLeod SD, West SK, Quigley HA, Fozard JL. A longitudinal relationship between intra-ocular and blood pressures. Invest Ophthalmol Vis Sci 199031 2361-2366.

12. Kaiser HJ, Flammer J, Graf T, Stumpfig D. Systemic blood pressure in glaucoma patients. Graefe's Arch Clin Exp Ophthalmol 1993231 677-680.

13. Klein BEK, Klein R, Knudtson MD. Intraocular pressure and systemic blood pressure: longitudinal perspective, the Beaver Dam Eye Study. Br J Ophthalmol 200589 284287.

14. Lee YW, Min WK, Chun S, Lee W, Kim Y, Chun SH, Park $\mathrm{H}$, Shin HB, Lee YK. The association between intraocular pressure and predictors of coronary heart disease risk in Koreans. J Korean Med Sci 200823 31-34.

15. Deokule S, Weinreb RN. Relationships among systemic blood pressure, intraocular pressure and open-angle glaucoma. Can J Ophthalmol 200843 302-307.

16. $\mathrm{Wu} \mathrm{SY,} \mathrm{Leske} \mathrm{MC.} \mathrm{Association} \mathrm{with} \mathrm{intraocular} \mathrm{pressure}$ in the Barbados eye study. Arch Ophthalmol 1997115 $1572-1576$. 